\title{
Mechanical cardiac remodeling and new-onset atrial fibrillation in long-term follow-up of subjects with chronic Chagas' disease
}

\author{
P.R. Benchimol-Barbosa ${ }^{1,2,3}$ and J. Barbosa-Filho ${ }^{3}$ \\ ${ }^{1}$ Serviço de Cardiologia, Hospital Universitário Pedro Ernesto, Universidade do Estado do Rio de Janeiro, \\ Rio de Janeiro, RJ, Brasil \\ 2Serviço de Cardiologia, Hospital Central Aristarcho Pessoa, Corpo de Bombeiros Militar do Estado do \\ Rio de Janeiro, Rio de Janeiro, RJ, Brasil \\ ${ }^{3}$ Instituto Nacional de Cardiologia, Universidade Gama Filho, Rio de Janeiro, RJ, Brasil \\ Correspondence to: P.R. Benchimol-Barbosa, Rua Pompeu Loureiro, 36/702, 22061-000 Rio de Janeiro, \\ RJ, Brasil \\ E-mail: ecgar@yahoo.com
}

\begin{abstract}
Atrial fibrillation (AF) affects subjects with Chagas' disease and is an indicator of poor prognosis. We investigated clinical, echocardiographic and electrocardiographic variables of Chagas' disease in a long-term longitudinal study as predictors of a new-onset AF episode lasting $>24 \mathrm{~h}$, nonfatal embolic stroke and cardiac death. Fifty adult outpatients (34 to 74 years old, $62 \%$ females) staged according to the Los Andes classification were enrolled. During a follow-up of (mean \pm SD) $84.2 \pm 39.0$ months, 9 subjects developed AF (incidence: $3.3 \pm 1.0 \% / y e a r), 5$ had nonfatal stroke (incidence: $1.3 \pm 1.0 \% / y e a r$ ), and nine died (mortality rate: $2.3 \pm 0.8 \%$ /year). The progression rate of left ventricular mass and left ventricular ejection fraction was significantly greater in subjects who experienced AF (16.4 \pm 20.0 g/year and $-8.6 \pm 7.6 \% / y e a r$, respectively) than in those who did not ( $8.2 \pm 8.4 \mathrm{~g} /$ year; $P=0.03$, and $-3.0 \pm 2.5 \%$ /year; $P=0.04$, respectively). In univariate analysis, left atrial diameter $\geq 3.2$ $\mathrm{cm}(P=0.002)$, pulmonary arterial hypertension $(P=0.035)$, frequent premature supraventricular and ventricular contraction counts/ $24 \mathrm{~h}(\mathrm{P}=0.005$ and $\mathrm{P}=0.007$, respectively), ventricular couplets $/ 24 \mathrm{~h}(\mathrm{P}=0.002)$, and ventricular tachycardia $(P=0.004)$ were long-term predictors of AF. P-wave signal-averaged ECG revealed a limited long-term predictive value for AF. In chronic Chagas' disease, large left atrial diameter, pulmonary arterial hypertension, frequent supraventricular and ventricular premature beats, and ventricular tachycardia are long-term predictors of AF. The rate of left ventricular mass enlargement and systolic function deterioration impact AF incidence in this population.
\end{abstract}

Key words: Chagas' disease; Cardiomyopathy; Atrial fibrillation; Cardiac death; Embolic stroke; Prognosis

Research partially supported by Faculdade de Ciências Médicas, Universidade do Estado do Rio de Janeiro (\#5152/2001), Rio de Janeiro, RJ, Brazil.

Received June 4, 2008. Accepted January 7, 2009

\section{Introduction}

Atrial fibrillation (AF) is the most common sustained arrhythmia amongst adults and bears an increased risk for morbid cardiovascular events, especially embolic stroke and heart failure $(1,2)$.

Chagas' disease is an anthropozoonosis fairly limited to the American continent and a foremost cause of chronic immune-mediated cardiomyopathy in Latin America (3-5). The systemic infection is generally acquired at an early age $(6,7)$ by vector transmission, although an acute form of parasitic transmission has been increasingly reported in affected countries (8), and may represent a noteworthy healthcare-related problem in Europe and the United States 
due to migration (9). Its insidious and asymptomatic course, ranging from 10 to 30 years, and eventual advance to myocardial dysfunction, occurring in up to $30 \%$ of infected subjects, represents a marker of unfavorable prognosis for cardiovascular morbidity and mortality (10-13). In this setting, persistent or permanent AF has been observed in up to one fifth of subjects bearing the chronic form (14) and represents a superimposed sign of poor prognosis $(7,8)$. The annual incidence of AF and other contributing factors, as well as time-varying functional and morphological characteristics of the heart eventually associated with the occurrence of $A F$ in this population, have not been fully investigated.

The purpose of the present study was to assess noninvasive clinical, echocardiographic, and 12-lead resting ECG, 24-h ambulatory ECG monitoring and signal averaged ECG parameters aiming at identifying predictive factors of persistent atrial fibrillation in subjects with Chagas' disease in a long-term longitudinal study.

\section{Patients and Methods}

In a longitudinal prospective study, a cohort of 50 consecutive subjects (34 to 74 years old; 31 females) with Chagas' disease followed up for at least 10 years before admission to the cardiomyopathy outpatient clinic of Pedro Ernesto University Hospital, Rio de Janeiro, RJ, Brazil, a tertiary care center, was enrolled from 1995 to 1996. Subjects were born in endemic regions of Minas Gerais and Bahia States of Brazil and Chagas' disease was confirmed before enrollment on the basis of positive serum tests, hemagglutination cruzipain-ELISA and indirect immunofluorescence. All subjects were referred to the arrhythmia unit with complaints of palpitations. Subjects were separated into three groups according to severity of heart involvement by the Los Andes classification (15): class I = 14 subjects, class II = 24 subjects, and class III = 12 subjects. Clinical and laboratory data were assessed during a personal interview and by review of the medical records. On admission, all subjects were in NYHA functional class I or II, had normal sinus rhythm and normal PR intervals. Exclusion criteria at enrollment were: any degree of atrioventricular block or non-sinus rhythm, previous documented acute coronary events (unstable angina or myocardial infarction), chronic obstructive pulmonary disease, rheumatic valvular heart disease, alcohol or illicit drug addiction, thyroid dysfunction or abnormal serum electrolytes. A treadmill stress test and/or coronary artery angiogram was indicated for selected subjects to rule out concomitant coronary artery disease. World Health Organization and Helsinki Treaty regulations reviewed in Venice
(1983) were followed.

\section{Clinical follow-up}

All subjects were monitored by the same team of physicians during the $>10$-year follow-up period. Medical visits were scheduled at the outpatient clinic at 3- to 6-month intervals and medications were prescribed at the discretion of the physician who performed the primary evaluation. Body weight varied $<2 \mathrm{~kg}$ during follow-up, and serum potassium varied from 3.5 to $5 \mathrm{mEq} / \mathrm{L}$. Mild to moderate systemic arterial hypertension (systolic arterial pressure ranging from 140 to $160 \mathrm{mmHg}$, or diastolic arterial pressure ranging from 90 to $115 \mathrm{mmHg}$ ) was observed in $41 \%$ of the subjects and was treated with converting enzyme inhibitors, diuretics, vasodilators, and/or beta-blockers at the discretion of the physician who performed the primary evaluation in order to reduce blood pressure levels to less than $140 / 90 \mathrm{mmHg}$. The endpoint was the first documented AF episode [(first AF); irregular non-sinus rhythm lasting $>24 \mathrm{~h}$ detected by either 24-h ambulatory ECG monitoring or 12-lead surface ECG]. The occurrence of embolic stroke (defined as sudden onset of focal deficit from the beginning and a brain computed tomography scan demonstrating ischemic infarct) and cardiac death (defined as intractable heart failure, sudden death, documented arrhythmic death or documented coronary occlusion) was also investigated in terms of temporal correlation to the occurrence of first AF during follow-up. It was hypothesized that persistent AF predicts a sustained arrhythmic episode and impacts survival. Besides regular outpatient follow-up, adverse events were actively tracked by review of medical records and by contact with relatives, in a regular basis, at least once a year.

\section{Resting surface ECG}

For each subject, standard resting 12-lead ECGs were recorded in the supine position (with simultaneous 3-lead acquisition) with a Cardimax ECAPS 122000 Compliant Electrocardiograph (Nihon-Kohden Co., Japan) on the same day of blood sample collection on admission. Electrocardiographic abnormalities were classified according to standard criteria for conduction disturbances (intraventricular and atrioventricular), chamber overload, and pathologic $Q$ waves. The electrocardiographic variables assessed in sinus rhythm were: P-wave duration (on lead II), PR interval (on lead II), QRS complex duration (the longest ventricular duration in precordial leads), presence of bundle branch block and/or left hemi-block, presence of pathological $Q$ waves (defined as the first $Q R S$ deflection >1-mm deep and $>0.04-m s$ wide), and left atrial overload ( $P$-wave duration in lead II $>110$ ms or Morris index in V1 $>4 \mathrm{mV} \times$ 
$\mathrm{ms})$. An independent observer blind to the study analyzed the electrocardiographic ( $2 \mathrm{~N}$ and $50 \mathrm{~mm} / \mathrm{s}$ ) records, automatically obtained from the electrocardiograph equipment. Subsequent 12-lead resting ECGs were recorded at each clinical visit in order to assess cardiac rhythm during follow-up.

\section{M-mode/2-D echocardiogram}

M-mode and 2-D echocardiograms were analyzed by a trained observer blind to the study protocol using an Apogee CX 200 apparatus (ATL, USA) with a 4-MHz broadband transducer. Echocardiographic parameters were assessed according to the standard procedures of the Section of Echocardiography of the Department of Cardiology, with special care taken to detect left ventricular (LV) apical aneurysms (16). The echocardiographic parameters assessed were LV ejection fraction (LVEF) calculated by the Teicholz method, M-mode LV end diastolic diameter, LV end systolic diameter, left atrial diameter (LAD), presence of pulmonary arterial hypertension (defined as maximal pulmonary arterial pressure $>30 \mathrm{mmHg}$, estimated by continuous-wave Doppler through tricuspid valve regurgitation), diastolic dysfunction, mild to moderate mitral regurgitation, LV mass (assessed by Devereux's formula) (17), and the presence of an apical aneurysm. No images suggestive of thrombus were detected in either atria or ventricles on a 2-D echocardiogram upon admission. Routine echocardiograms were performed in order to track changes in LV mass and LVEF during follow-up.

\section{4-h ambulatory ECG monitoring}

Twenty-four-hour ambulatory ECG monitoring was performed using a three-channel DMS cassette-tape recorder and carefully analyzed using the Del-Mar Avionics Strata Scan System (Del Mar Avionics, USA) by a trained observer blind to the study in order to assess the presence of ventricular arrhythmia and atrioventricular conduction disturbances. Variables assessed in the 24-h ambulatory ECG were: i) isolated premature supraventricular contractions (PSVC/24 h), ii) nonsustained supraventricular tachycardia defined as a sequence of three of more supraventricular ectopic beats $/ 24 \mathrm{~h}$, iii) isolated premature ventricular contractions (PVC/24 h), iv) coupled ventricular premature complexes (couplets/24 h), v) polymorphic PVC (PVCs with more than one morphology), and vi) ventricular tachycardia (VT) episodes (heart rate $>100 \mathrm{bpm}$, QRS duration $>120 \mathrm{~ms}$, and atrioventricular dissociation). Sustained VT was defined as episodes of VT lasting $30 \mathrm{~s}$ or more, with non-sustained VT being characterized by different values. During follow-up, 24-h ambulatory ECG was undertaken at the discretion of the attending physician in order to assess cardiac rhythm and arrhythmia. Tape recordings were successfully analyzed immediately after acquisition and both normal rhythm and arrhythmia episodes were appropriately assessed.

\section{Signal-averaged electrocardiogram}

A signal-averaged electrocardiogram (SAECG) was acquired in sinus rhythm with a Predictor IIc apparatus (ART Inc., USA) using Frank XYZ orthogonal leads and QRS-triggered coherent-averaged up to the noise level of $0.3 \mu \mathrm{V}$. SAECGs were analyzed in both time and frequency domains using a previously reported technique $(18,19)$ by an independent observer blind to the study subjects. SAECG was analyzed on the vector magnitude in the time domain and parameters were: duration of the filtered P-wave (ms), root-mean-squared voltage of the terminal $20 \mathrm{~ms}$ of signal-averaged $\mathrm{P}$-wave $(\mu \mathrm{V})$, and rootmean-squared voltage of the total signal-averaged $\mathrm{P}$-wave $(\mu \mathrm{V})$.

Treadmill stress test and coronary artery angiography

Selected subjects underwent a treadmill stress test in order to evaluate the presence of coronary artery disease. Five subjects underwent coronary angiography and ventriculography after an inconclusive test and all demonstrated normal coronary artery anatomy. An apical aneurysm was demonstrated in 3 subjects during ventriculography.

Clinical, echocardiographic, electrocardiographic, 24-h ambulatory ECG, and SAECG variables grouped according to the Los Andes classification are presented in Table 1.

\section{Statistical analysis}

Categorical variables are reported as ratio or percentage, and continuous variables as mean $\pm \mathrm{SD}$. Categorical variables were compared by the Yates-corrected chi-square test for contingency tables or by the Fisher exact test when appropriate. Continuous variables were compared by the Mann-Whitney test. Kruskal-Wallis ANOVA was used for inter-group comparison.

In univariate analyses, the relative risk (RR) for first AF endpoint and respective $95 \%$ confidence interval $(\mathrm{Cl})$ were assessed for all significant variables. For continuous variables, RR was estimated at the optimal dichotomization threshold calculated by ROC analysis. Significant variables entered a univariate Cox proportional-hazard regression model to determine first AF risk. The output parameter of the model was analyzed as hazard ratio. Kaplan-Meiers event-free survival function was assessed in significant variables in the model.

General exploratory plots are presented in box-andwhisker format (median and inter-quartile range). Cardiac 
remodeling was assessed retrospectively according to endpoint achieved as: i) the difference between the last (end of follow-up) and initial (admission) echocardiographic variable values, and ii) the average variation rate, defined as the value in (i) divided by total follow-up time to endpoint of the respective variable.

At the end of follow-up, $3 \pm 1$ echocardiograms, $2 \pm 1$ 24-h episodes of ambulatory ECG monitoring and $19 \pm 4$ resting ECG tracings per subject were analyzed. No significant differences in the number of echocardiograms or 24-h ambulatory ECG monitoring requests by the primary assistance were observed between those who did not experience and those who experienced first AF (echocardiogram: $4 \pm 1$ vs $3 \pm 1$ exams; $P=0.11$, and $24-\mathrm{h}$ ambu-

Table 1. Clinical, electrocardiographic and echocardiographic parameters of the subjects on admission.

\begin{tabular}{|c|c|c|c|c|}
\hline & \multicolumn{3}{|c|}{ Los Andes classification } & \multirow[t]{2}{*}{$\mathrm{P}$} \\
\hline & Class I & Class II & Class III & \\
\hline \multicolumn{5}{|l|}{ Clinical variables } \\
\hline $\mathrm{N}$ & 14 & 24 & 12 & - \\
\hline Age (years) & $52.6 \pm 7.8$ & $51.5 \pm 9.9$ & $49.8 \pm 10.6$ & - \\
\hline Gender (female/male) & $9 / 5$ & $19 / 5$ & $7 / 5$ & - \\
\hline Systemic arterial hypertension & $42.9 \%$ & $45.5 \%$ & $25.0 \%$ & - \\
\hline NYHA functional class $>1$ & $0 \%$ & $0 \%$ & $33.3 \%$ & 0.001 \\
\hline \multicolumn{5}{|l|}{ Resting 12-lead electrocardiogram } \\
\hline P-wave duration (ms) & $90.0 \pm 17.1$ & $91.3 \pm 17.0$ & $89.2 \pm 22.7$ & - \\
\hline QRS duration (ms) & $80.0 \pm 7.8$ & $127.5 \pm 24.7$ & $121.7 \pm 31.6$ & $<0.001$ \\
\hline Left atrial overload & $7.1 \%$ & $12.5 \%$ & $16.7 \%$ & - \\
\hline Left bundle branch block ${ }^{a}$ & $0 \%$ & $16.7 \%$ & $16.7 \%$ & - \\
\hline Right bundle branch block ${ }^{a}$ & $0 \%$ & $70.8 \%$ & $41.7 \%$ & 0.001 \\
\hline Left anterior hemi-block & $0 \%$ & $41.7 \%$ & $50.0 \%$ & 0.009 \\
\hline Inactive area & $0 \%$ & $25.0 \%$ & $41.7 \%$ & 0.03 \\
\hline \multicolumn{5}{|l|}{ M-mode and 2-D mode echocardiogram } \\
\hline $\operatorname{LAD}(\mathrm{mm})$ & $3.5 \pm 0.5$ & $3.5 \pm 0.6$ & $3.9 \pm 0.7$ & - \\
\hline LVESD (mm) & $3.1 \pm 0.7$ & $2.8 \pm 0.6$ & $5.0 \pm 0.9$ & $<0.001$ \\
\hline LVEDD (mm) & $5.0 \pm 0.7$ & $4.8 \pm 0.7$ & $6.1 \pm 0.9$ & 0.001 \\
\hline LVEF & $71.7 \pm 9.6$ & $76.5 \pm 9.1$ & $36.3 \pm 11.4$ & $<0.001$ \\
\hline Left ventricular mass $(\mathrm{g})$ & $157.0 \pm 54.7$ & $150.0 \pm 57.0$ & $217.2 \pm 69.5$ & 0.015 \\
\hline Type I diastolic dysfunction & $28.0 \%$ & $48.0 \%$ & $24.0 \%$ & 0.02 \\
\hline Mitral valve regurgitation & $28.0 \%$ & $48.0 \%$ & $24.0 \%$ & 0.02 \\
\hline Pulmonary arterial hypertension ${ }^{b}$ & $28.0 \%$ & $48.0 \%$ & $24.0 \%$ & 0.02 \\
\hline Apical aneurysm & $7.7 \%$ & $25.0 \%$ & $18.2 \%$ & - \\
\hline \multicolumn{5}{|l|}{ 24-h ambulatory electrocardiogram } \\
\hline Isolated PSVC (count/24 h) & $44 \pm 64$ & $598 \pm 1605$ & $2156 \pm 3428$ & 0.03 \\
\hline NSupVT & $28.6 \%$ & $46.9 \%$ & $24.5 \%$ & 0.04 \\
\hline Isolated PVC (count/24 h) & $2029 \pm 4116$ & $602 \pm 1008$ & $7505 \pm 8246$ & 0.002 \\
\hline Couplets (count/24 h) & $16 \pm 39$ & $11 \pm 25$ & $457 \pm 730$ & $<0.001$ \\
\hline Polymorphic PVC & $50.0 \%$ & $50.0 \%$ & $91.7 \%$ & 0.03 \\
\hline NSVT & $14.3 \%$ & $12.5 \%$ & $58.3 \%$ & 0.006 \\
\hline \multicolumn{5}{|l|}{ Signal-averaged electrocardiogram } \\
\hline Dur-P (ms) & $125.3 \pm 19.1$ & $124.8 \pm 15.8$ & $121.0 \pm 24.8$ & - \\
\hline RMS20 ( $\mu \mathrm{v})$ & $6.2 \pm 3.6$ & $4.5 \pm 2.5$ & $5.7 \pm 2.7$ & - \\
\hline $\operatorname{RMSP}(\mu \mathrm{v})$ & $9.4 \pm 2.9$ & $8.9 \pm 4.1$ & $8.4 \pm 2.6$ & - \\
\hline
\end{tabular}

Data are reported as means $\pm S D$ or percent. NYHA $=$ New York Heart Association; LAD = left atrial diameter; LVESD = left ventricular end systolic diameter; LVEDD = left ventricular end diastolic diameter; LVEF = left ventricular ejection fraction; PSVC = premature supraventricular contraction; NSupVT = nonsustained supraventricular tachycardia; $\mathrm{PVC}=$ premature ventricular contraction; NSVT = nonsustained ventricular tachycardia; Dur-P = P-wave signal-averaged duration; RMS20 = root-mean-squared voltage of the last 20

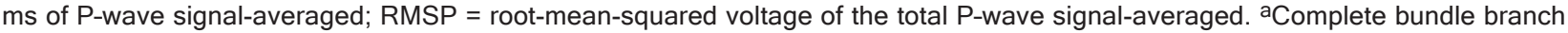
block - QRS duration $>120 \mathrm{~ms}$. b Pulmonary arterial hypertension: pulmonary arterial pressure $>30 \mathrm{mmHg}$ (see text for details). $\mathrm{P}$ values $<0.05$ are reported (Yates-corrected chi-square test or Kruskal-Wallis ANOVA, when appropriate). 
latory ECG: $2 \pm 1$ vs $2 \pm 1$ exams; $\mathrm{P}=0.95$, respectively).

A post hoc power (1- $\beta$ error level) calculation was carried out on variables that achieved statistical significance. A univariate power analysis based on derivation of Fieiss' formula (20) was carried out on the variable that demonstrated the highest RR. Non-exposed and exposed populations were arbitrarily defined as class I and classes II plus III, respectively. The alpha error level was set at 0.05 . The software packages used for data analysis were MS Excel 2000 (Microsoft Corporation, USA), Medcalc version 9 (Medcalc Software, Belgium), Epi-Info 6.04 (Center for Disease Control, USA), and Statgraphics version 5.1 Plus (Manugistic Inc., USA).

\section{Results}

\section{Clinical follow-up}

The overall mean follow-up time was $84.2 \pm 39.0$ months, ranging from 6 to 120 months. During follow-up, first $\mathrm{AF}$ was documented in 9 subjects (incidence: $3.3 \pm$ $1.0 \% /$ year), 5 subjects developed nonfatal embolic stroke (incidence: $1.3 \pm 1.0 \% /$ year) and 9 subjects died (mortality rate: $2.3 \pm 0.8 \%$ /year). Average heart rate registered on surface ECG during AF episodes ranged from 90 to 120 bpm. Among subjects who experienced first AF (overall follow-up time to event: $45.5 \pm 21.3$ months), 2 subjects (22.2\%; RR $=3.0 ; 95 \% \mathrm{Cl}: 0.6-15.6 ; \mathrm{P}=0.22)$ had subsequent nonfatal embolic stroke (median 8.5 months after first $A F)$, and 4 subjects $(44.4 \%$; $R R=3.6 ; 95 \% \mathrm{Cl}: 1.2-$ $10.9) ; P=0.039$ ) subsequently died during follow-up (median 25.9 months after first AF). Relative risk of stroke for cardiac death was $1.1(95 \% \mathrm{Cl}$ : 0.2-7.2; $\mathrm{P}=0.62)$.

Overall variation in LV mass and LVEF between admission and end of follow-up were $75.2 \pm 50.2 \mathrm{~g}(\mathrm{P}<0.001)$ and $-14.6 \pm 10.4 \%(P<0.001)$, respectively. Changes in LV mass and LVEF parameters assessed during follow-up in subjects who experienced and did not experience AF are presented in Figure 1A and B. LV mass significantly increased both in those who experienced and did not experience AF (Figure 1A). However, the average variation rate of $L V$ mass was significantly greater in the group that experienced AF (16.4 $\pm 20.0 \mathrm{~g} /$ year) compared to the one that did not $(8.2 \pm 8.4 \mathrm{~g} /$ year; $\mathrm{P}=0.03)$. Correspondingly, LVEF decreased more rapidly $(-8.6 \pm 7.6 \% / y e a r)$ in the group that experienced $\mathrm{AF}$ as compared to the one that did not $(-3.0 \pm 2.5 \% /$ year; $\mathrm{P}=0.04$; Figure $1 \mathrm{~B})$.

According to the Los Andes classification, first AF was observed in $14.3 \%$ of patients in class I, $16.7 \%$ in class II, and $25.5 \%$ in class III $(P=0.76)$. The overall prevalence of stroke after the first AF was $14.3 \%$ in class I, $8.3 \%$ in class
A

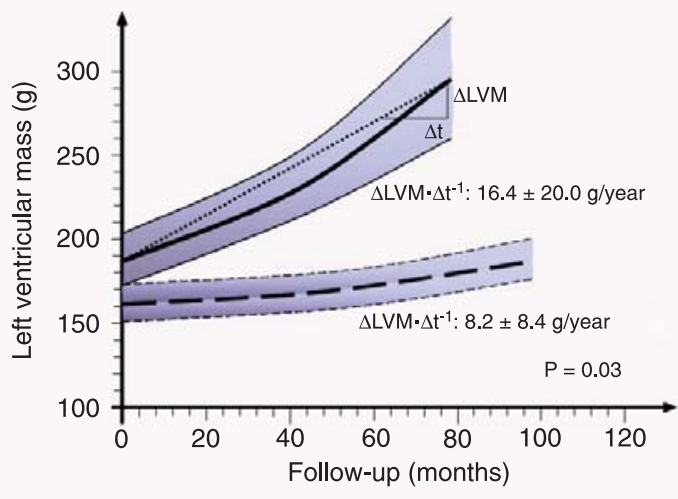

\begin{tabular}{l|c|c|c}
\hline & Admission & End of follow-up & $P$ \\
\hline No AF (---) & $161 \pm 64$ & $186 \pm 63$ & 0.001 \\
\hline AF (-) & $186 \pm 62$ & $297 \pm 130$ & 0.01 \\
\hline P & 0.17 & 0.01 & - \\
\hline
\end{tabular}

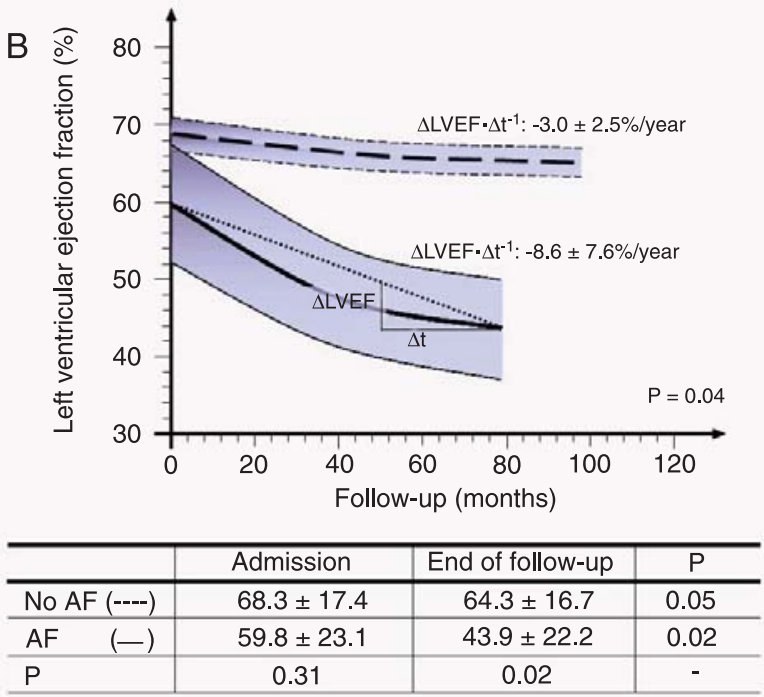

Figure 1. Projected temporal variation in geometric and functional parameters during follow-up according to the occurrence of atrial fibrillation (AF). For each graph, lines are average echocardiographic data trend and boundaries are one standard deviation. Dashed line, No AF group; solid line, AF group. The paired test $P$ value is presented at the bottom of the Table. Data are reported as means $\pm \mathrm{SD}$. A, Left ventricular mass (LVM). Note the significant increase in LVM in both the AF group and No AF group (Table). However, inter-group average LVM variation rate showed significant differences $(P=0.03)$. $\Delta L V M \cdot \Delta t^{-1}$, average left ventricular mass variation rate. $B$, Left ventricular ejection fraction (LVEF) during follow-up. Note the significant reduction in LVEF observed in the AF group compared to No AF group. Inter-group average LVEF variation rate showed statistically significant differences $(P=0.04$; see text for details). $\Delta \mathrm{LVEF} \cdot \Delta \mathrm{t}^{-1}$, average left ventricular ejection fraction variation rate. 
II, and $8.3 \%$ class III $(P=0.82)$. The average annual variation rate in LV mass and LVEF is presented in Figure $2 \mathrm{~A}$ and $\mathrm{B}$. Both variables showed non-significant differences across the Los Andes groups.

At the end of follow-up, there was a trend $(P=0.07)$ toward a lower LVEF among subjects who experienced first AF and died $(39.3 \pm 34.8 \%)$ as compared to those who experienced first AF and survived (59.8 $\pm 17.1 \%)$. However, regarding first AF occurrence, a non-significant difference in LVEF was observed in subjects who did not experience AF and died $(29.7 \pm 8.4 \%$; $P=0.53)$, as well as in those who did not experience AF and survived (66.5 \pm $13.5 \% ; P=0.14)$, respectively.

Univariate analyses of clinical, echocardiogram, and resting 12-lead, 24-h ambulatory monitoring and SAECG as predictors for persistent $\mathrm{AF}$

Univariate analyses of significant variables of 12-lead resting ECG, echocardiogram, 24-h ambulatory ECG monitoring, and SAECG variables are summarized in Table 2. The prevalence of systemic arterial hypertension did not differ significantly between those who experienced (11.1\%) and did not experience (46.2\%; $\mathrm{P}=0.07) \mathrm{AF}$ during followup, as was also the case for the use of ACE inhibitors, betablockers and diuretics. Age and gender distribution did not differ between those who did and did not develop first AF $(P=0.15$ and $P=0.99$, respectively).

Figure 2. Box-and-whisker plots of average annual variation in parameters according to Los Andes classes I, II and III during follow-up. Kruskal-Wallis test $P$ value is displayed on the top of the boxes. The small cross-shaped marks represent the mean values. Statistics on the top are reported as mean $\pm \mathrm{SD}$. $A$, Left ventricular mass (LVM). Note that the average annual variation in $\mathrm{LVM}\left[\Delta \mathrm{LVM} \cdot \Delta \mathrm{t}^{-1}\right.$ (g/year)] observed across the top of each of the Los Andes classes was not statistically significant. $B$, Left ventricular ejection fraction (LVEF) during follow-up. Note that the average annual variation in LVEF [ $\Delta \mathrm{LVEF} \cdot \Delta \mathrm{t}^{-1}$ (\%/year)], means $\pm \mathrm{SD}$, observed across the top of each of the Los Andes classes was not statistically significant (see text for details).
Significant variables were: i) echocardiographic parameters (Table 2$)$ : $L A D(P=0.014)$, pulmonary arterial hypertension ( $\mathrm{RR}=7.2 ; 95 \% \mathrm{Cl}: 2.8-18.4 ; \mathrm{P}=0.002)$, ii) 24-h ambulatory ECG: isolated-PSVC counts/24 h ( $P$ $=0.016)$, isolated-PVC counts $/ 24 \mathrm{~h}(\mathrm{P}=0.03)$, couplet counts $/ 24$ h $(P<0.02)$, and VT $(R R=4.0 ; 95 \% C l: 1.3-$ 12.4; $P=0.026)$.

Clinical (age, gender, NYHA class, Los Andes class) and P-wave SAECG variables demonstrated a nonsignificant predictive value for first AF (Table 2).

The optimal thresholds assessed by ROC analysis of significant variables were: $L A D \geq 3.2 \mathrm{~mm}$ (100.0\% sensitivity, $43.9 \%$ specificity; $\mathrm{RR}=1.6 ; 95 \% \mathrm{Cl}: 1.1-2.3)$, isolatedPSVC $\geq 243$ counts 24 h $(66.7 \%$ sensitivity, $82.5 \%$ specificity; $\mathrm{RR}=5.7 ; 95 \% \mathrm{Cl}: 1.7-19.5), \geq 2973$ isolated-PVC counts/24 h (66.7\% sensitivity, $85.4 \%$ specificity; RR = 5.7; $95 \% \mathrm{Cl}: 1.7-19.5)$ and $\geq 27$ couplet counts/24 h $(66.7 \%$ sensitivity, $82.9 \%$ specificity; $\mathrm{RR}=5.1 ; 95 \% \mathrm{Cl}$ : $1.5-17.8)$. Univariate Cox proportional-hazard model analysis is presented in Table 3.

The average variation rate in LVEF in the presence of pulmonary arterial hypertension (-10.2 $\pm 7.6 \% / y e a r)$ was significantly lower than in patients who did not have pulmonary hypertension $(-3.0 \pm 2.4 \%$ /year; $P=0.009)$. No differences in LVEF variation rate was observed regarding isolated-PVC counts/24 h (<2973 counts/24h: $-4.1 \pm 5.1 \%$ / year vs $\geq 2973$ counts/24 h: $-4.8 \pm 2.3 \%$ /year; $P=0.16)$. No
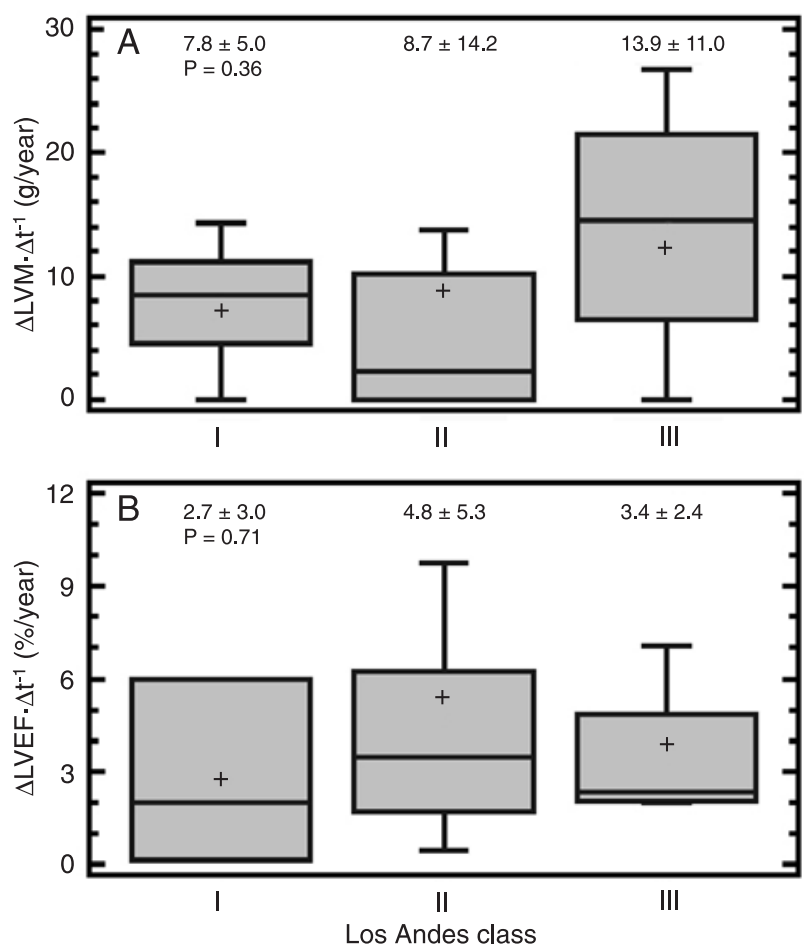

Los Andes class 
significant differences were observed regarding average variation rate in LV mass, as well. Kaplan-Meier curves for significant univariate predictors of first AF are illustrated in Figure 3.

\section{Post hoc statistical power analysis}

Statistical power (1- $\beta$ ) was calculated post hoc for significant variables in univariate analysis, at a 0.05 alpha error level and based on optimal cut-off values for continu-

Table 2. Clinical, electrocardiographic, and echocardiographic variables assessed on admission as a function of clinical outcome during follow-up.

\begin{tabular}{|c|c|c|c|}
\hline & AF group & No AF group & $P$ \\
\hline \multicolumn{4}{|l|}{ Clinical variables } \\
\hline $\mathrm{N}$ & 9 & 41 & - \\
\hline Age (years) & $55.4 \pm 10.2$ & $50.5 \pm 9.1$ & - \\
\hline Gender (female/male) & $6 / 3$ & $19 / 12$ & - \\
\hline Systemic arterial hypertension & $11.1 \%$ & $46.2 \%$ & - \\
\hline NYHA functional class $>1$ & $22.2 \%$ & $4.9 \%$ & - \\
\hline Los Andes class III & $33.3 \%$ & $22.0 \%$ & - \\
\hline \multicolumn{4}{|l|}{ Resting 12-lead electrocardiogram } \\
\hline P-wave duration (ms) & $80.0 \pm 22.4$ & $92.7 \pm 16.6$ & - \\
\hline QRS duration (ms) & $122.2 \pm 35.6$ & $110.7 \pm 30.0$ & - \\
\hline Left atrial overload & $11.1 \%$ & $12.2 \%$ & - \\
\hline Left bundle branch blocka & $22.2 \%$ & $9.8 \%$ & - \\
\hline Right bundle branch block ${ }^{a}$ & $55.6 \%$ & $41.5 \%$ & - \\
\hline Left anterior hemi-block & $44.4 \%$ & $29.3 \%$ & - \\
\hline Inactive area & $11.1 \%$ & $24.4 \%$ & - \\
\hline \multicolumn{4}{|l|}{ M-mode and 2-D mode echocardiogram } \\
\hline LAD $(\mathrm{mm})$ & $4.0 \pm 0.6$ & $3.5 \pm 0.6$ & 0.014 \\
\hline LVESD (mm) & $3.8 \pm 1.4$ & $3.2 \pm 1.0$ & - \\
\hline LVEDD (mm) & $5.5 \pm 1.1$ & $5.0 \pm 0.8$ & - \\
\hline LVEF & $59.8 \pm 23.1$ & $68.3 \pm 17.4$ & - \\
\hline Left ventricular mass $(\mathrm{g})$ & $186.1 \pm 62.0$ & $161.4 \pm 64.0$ & - \\
\hline Type I diastolic dysfunction & $11.1 \%$ & $24.4 \%$ & - \\
\hline Mitral valve regurgitation & $77.8 \%$ & $46.3 \%$ & - \\
\hline Pulmonary arterial hypertension ${ }^{b}$ & $44.4 \%$ & $2.4 \%$ & 0.002 \\
\hline Apical aneurysm & $22.2 \%$ & $20.5 \%$ & - \\
\hline \multicolumn{4}{|l|}{ 24-h ambulatory electrocardiogram } \\
\hline Isolated PSVC (count/24 h) & $1729 \pm 2635$ & $617 \pm 1977$ & 0.016 \\
\hline NSupVT & $66.7 \%$ & $42.5 \%$ & - \\
\hline Isolated PVC (count/24 h) & $6269 \pm 7953$ & $1866 \pm 4276$ & 0.03 \\
\hline Couplets (count/24 h) & $377 \pm 765$ & $63 \pm 239$ & 0.02 \\
\hline Polymorphic PVC & $77.8 \%$ & $61.0 \%$ & - \\
\hline NSVT & $55.6 \%$ & $17.1 \%$ & 0.03 \\
\hline \multicolumn{4}{|l|}{ Signal-averaged electrocardiogram } \\
\hline Dur-P (ms) & $128.9 \pm 24.5$ & $123.0 \pm 17.6$ & - \\
\hline RMS20 ( $\mu \mathrm{v})$ & $5.3 \pm 3.0$ & $5.3 \pm 2.9$ & - \\
\hline RMSP $(\mu v)$ & $9.2 \pm 5.3$ & $8.9 \pm 2.9$ & - \\
\hline
\end{tabular}

Data are reported as means \pm SD or percent. $A F=$ atrial fibrillation; NYHA = New York Heart Association; LAD $=$ left atrial diameter; LVESD = left ventricular end systolic diameter; LVEDD = left ventricular end diastolic diameter; LVEF = left ventricular ejection fraction; PSVC = premature supraventricular contraction; NSupVT = nonsustained supraventricular tachycardia; PVC = premature ventricular contraction; NSVT = nonsustained ventricular tachycardia; Dur-P = P-wave signal-averaged duration; RMS20 = root-mean-squared voltage of the last $20 \mathrm{~ms}$ of $\mathrm{P}$-wave signal-averaged; RMSP = root-mean-squared voltage of the total P-wave signalaveraged. ${ }^{a}$ Complete bundle branch block - QRS duration $>120$ ms. bPulmonary arterial hypertension: pulmonary arterial pressure $>30 \mathrm{mmHg}$ (see text for details). $P$ values $<0.05$ are reported [Yates-corrected chisquare (Fisher exact) or Mann-Whitney tests, when appropriate]. 
Table 3. Univariate Cox proportional-hazard regression model analysis of significant predictors for atrial fibrillation during follow-up.

\begin{tabular}{lccrrrr} 
Variable & $\beta$ coefficient & SEM & $\chi^{2}$ test & $\mathrm{P}$ & $\mathrm{HR}$ & $95 \% \mathrm{Cl}$ \\
\hline LAD $\geq 3.2 \mathrm{~cm}$ & 13.9 & 0.9 & 9.7 & 0.002 & 10 & $1.01-10.5$ \\
Pulmonary arterial hypertension & 2.2 & 0.7 & 8.2 & 0.004 & 8.9 & $2.3-33.6$ \\
Isolated PSVC $\geq 243$ (count/24 h) & 1.9 & 0.7 & 7.9 & 0.005 & 6.8 & $1.7-27.1$ \\
Isolated PVC $\geq 2973$ (count/24 h) & 2.4 & 0.7 & 11.5 & 0.001 & 11.1 & $2.7-45.4$ \\
Ventricular couplets $\geq 27$ (count/24 h) & 2.2 & 0.7 & 10.1 & 0.002 & 9.1 & $2.3-38.8$ \\
NSVT & 2.1 & 0.7 & 8.4 & 0.004 & 7.8 & $2.1-29.6$ \\
\hline
\end{tabular}

$\beta$ coefficient $=$ coefficient estimate of the variable SEM = standard error of the estimate; $\mathrm{HR}=$ hazard ratio; $\mathrm{Cl}=$ confidence interval; $\mathrm{PVC}=$ premature ventricular contraction; $\mathrm{PSVC}=$ premature supraventricular contraction; $L A D=$ left atrial diameter; NSVT = nonsustained ventricular tachycardia; pulmonary arterial hypertension (pulmonary arterial pressure $>30 \mathrm{mmHg}$ ) assessed on admission. Cut-off thresholds were determined by ROC analysis (see Methods for details).
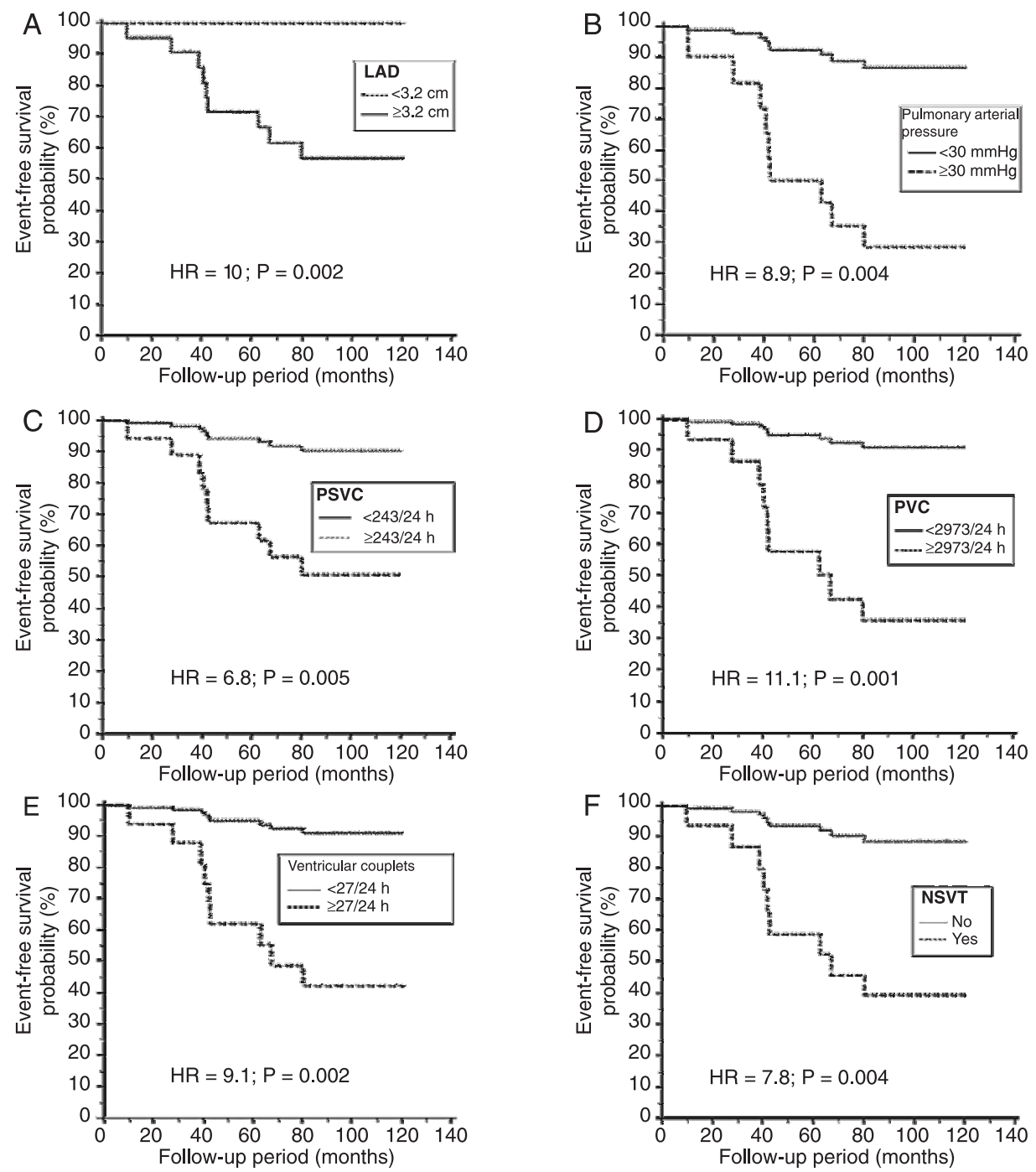

Figure 3. Kaplan-Meier event-free survival probability function to first persistent atrial fibrillation episode in subjects grouped according to left atrial diameter ( $\mathrm{LAD}, \mathrm{A})$; pulmonary arterial pressure $(\mathrm{B})$; isolated premature supraventricular contractions (PSVC), counts/24 h (C); isolated premature ventricular contractions (PVC), counts/24 h (D); ventricular couplets, counts/24 h (E); nonsustained ventricular tachycardia (NSVT, F) assessed on admission. P values indicate statistical significance according to hazard ratio $(\mathrm{HR})$ in the univariate Cox proportional-hazard model (see text for details). 
ous variables. Analyses yielded the following statistical power estimates: $41.5 \%(95 \% \mathrm{Cl}: 39.5-43.5 \%)$ for LAD $\geq 3.2 \mathrm{~cm}, 98 \%$ (95\% Cl: $97.4-98.6 \%)$ for pulmonary hypertension, $98.5 \%(95 \% \mathrm{Cl}: 98.0-99.0 \%)$ for isolated-PVC $\geq 2973$ counts $/ 24$ h, $98.9 \%$ (95\%Cl: $98.5-99.3 \%)$ for couplets $\geq 27$ counts $/ 24 \mathrm{~h}$, and $88.4 \%$ (95\%Cl: $87.1-89.7 \%)$ for nonsustained ventricular taquicardia.

In particular, regarding pulmonary arterial hypertension (PAH), there was a 2.8 RR for first AF (lower boundary of $95 \% \mathrm{Cl}$ for respective $\mathrm{RR}$ ), assuming a 2.6 non-exposed-to-exposed ratio and $19.5 \%$ incidence among nonexposed (class I), an overall 92.9\% (95\%Cl: 82.1-95.6\%) power for first AF prediction was achieved.

\section{Discussion}

In the present study, clinical, electrocardiographic, and echocardiographic parameters were assessed during longterm follow-up aiming at investigating predictors for the first documented episode of AF lasting $>24 \mathrm{~h}$ in subjects with Chagas' disease, and further to evaluate mechanical cardiac remodeling during follow-up eventually associated with development of this arrhythmia. Although AF is the most common arrhythmia in the general population and therapeutic strategies to circumvent related adverse events have been extensively explored so far, the identification of potential disease-oriented predisposing factors such as those occurring in systemic inflammatory diseases affecting the heart, like Chagas' disease, may impact both medical assistance and patient quality of life.

In a previous communication, we have shown that a larger left atrial diameter is a predictor of new onset AF during follow-up in chronic Chagas' heart disease, although the variation rate of left atrial diameter did not differ between those who experienced and those who did not experience new onset AF (21). To the best of our knowledge, the present study is the first long-term follow-up study to assess cardiac remodeling and noninvasive predictors of an AF episode in subjects with chronic Chagas' disease.

Among subjects with Chagas' disease assisted at outpatient clinics of our hospital from 1985 and 1996, those with complaints of palpitations referred to the arrhythmia unit, representing a $9.1 \%$ prevalence in our clinics, and with normal atrial-ventricular conduction composed the cohort of the present study. It is particularly interesting to note that palpitations, a symptom commonly related to arrhythmia, have been considered to be a possible indication of adverse outcome in Chagas' disease (22), and conduction delay, on the other hand, reported in 5.8 to $20.2 \%$ of subjects with chronic Chagas' cardiomyopathy
(23), has been regarded as a degenerative disturbance and a consequence of conduction system fibrosis (24).

The identification of predictive factors associated with the risk for $A F$ has been a general concern since the Framingham study, and several factors have been associated with a higher incidence of AF in the general population $(18,25-30)$. In the present study, the prevalence of systemic arterial hypertension did not differ across Los Andes groups on admission (Table 1) and neither the distribution of systemic hypertension nor drug therapy to control blood pressure among subjects who experienced and did not experience AF was statistically different at the end of follow-up. This indicates a direct impact of chagasic cardiomyopathy itself on AF incidence in addition to other known risk factors. K-M survival curves for systolic arterial hypertension diagnosed on admission due to first AF are presented in Figure 4. A limited number of studies have assessed AF either transversally or in longitudinal studies on Chagas' disease. Prata et al. (14) found a 5.5\% prevalence of AF in chagasic subjects younger than 60 , rising to $19.6 \%$ in older ones. lanni et al. (31) assessed prospectively a group of subjects with the undetermined form of Chagas' disease and found a very low incidence of the arrhythmia in a long-term follow-up $(0.6 \%$ in 177 months of follow-up). In the present study, a high incidence of first AF was observed, which corresponded to $18.0 \%$ prevalence at the end of follow-up. These estimates indicate that subjects with Chagas' disease have both a higher inci-

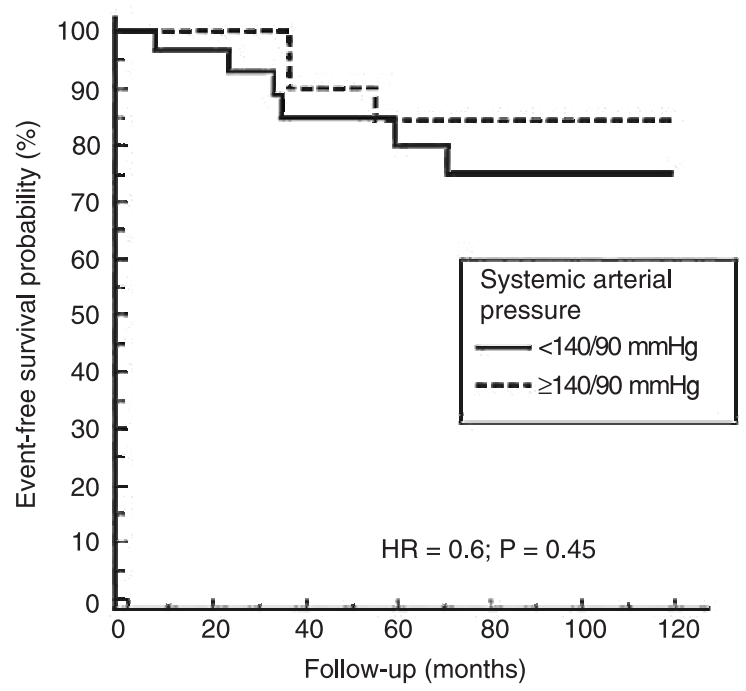

Figure 4. Kaplan-Meier event-free probability function to first persistent atrial fibrillation (first AF) episode detected in subjects grouped according to the diagnosis of systemic arterial hypertension (SAH) on admission. Hazard ratio (HR) of SAH for first AF was $0.6(95 \% \mathrm{Cl}: 0.2-2.3 ; \mathrm{P}=0.45$; log-rank test). 
dence and a higher prevalence of AF than age-matched subjects from the general population (32).

In Chagas' disease, AF has been considered a marker of poor prognosis of cardiac death (33), and other concomitant disease-related markers impose a higher risk for embolic stroke (34). In the present study, AF was associated with a non-significant increase in stroke risk. It is noteworthy that, at the end of follow-up, the prevalence of documented AF episodes and of stroke was not significantly different across Los Andes groups. On the other hand, AF did increase the subsequent risk for cardiac death. The explanation for the former observation may rely on the fact that, although AF represents an independent risk for embolic stroke, other conditions associated with Chagas' disease may increase the risk in addition to $\mathrm{AF}$ itself $(34,35)$.

Permanent AF has been regarded as a potentially reversible cause of LV systolic dysfunction due to both shortening of the systolic interval and loss of the atrial contraction-mediated Frank-Starling mechanism (36). In the present study, at the end of follow-up there was a trend toward a lower LVEF in subjects who experienced first AF and died as compared to those who experienced first AF and survived. However, regarding survival, a non-significant difference in LVEF was observed in subjects who did not experience AF and died vs subjects who experienced first $A F$ and died $(P=0.53)$, as well as in subjects who did not experience AF and survived vs subjects who experienced first $A F$ and survived $(P=0.14)$. These findings lead us to consider that AF development had a modest impact on further systolic function deterioration in this population. Thus, it was hypothesized that in Chagas' disease the progression of LV dysfunction and AF are a sequence in the natural history of the disease. The rapid deterioration of LV systolic function is related to a poorer prognosis $(37,38)$, eventually leading to AF and further depressing pump function, heralding cardiac failure and death, in this setting. A mathematical formulation describing both the structural and functional progression of heart involvement in Chagas' disease has already been proposed (38) and was applied to describe time-varying changes in LVEF and LV mass during follow-up.

In the present study, left atrial diameter, PAH and frequent supraventricular and ventricular ectopic beats assessed on admission were predictors of first AF during follow-up. In particular, the higher prevalence of $\mathrm{PAH}$ in this population was associated with the presence of both diastolic dysfunction and mitral regurgitation. Mechanistically, either diastolic dysfunction or mitral regurgitation influences pulmonary venous pressure and thus prospectively affects $\mathrm{PAH}$. In fact, across the Los Andes groups the higher prevalence of PAH was associated with a higher prevalence of both diastolic dysfunction and mitral regurgitation in the same group. Although the prognostic value of $\mathrm{PAH}$ for AF has not been extensively explored, Funk et al. (30) have demonstrated that PAH represents an independent risk factor for AF after cardiac surgery and hospital discharge. On the other hand, frequent PVCs have been considered to be a sign of poor prognosis and to predict development of heart failure (39). Thus, both PAH and PVC may have impacted the incidence of first AF by different mechanisms.

The signal-averaged electrocardiogram is a valuable prognostic tool for either paroxysmal or persistent AF (18, 27,40). In the present study, however, P-SAECG did not demonstrate a significant predictive value for first AF. We considered that non-significant predictive value observed in the present study to be due, at least in part, to the fact that all subjects but one showed normal P-SAECG duration on admission. The normality cut-off for P-wave SAECG vector magnitude duration is $155 \mathrm{~ms}$ and only one subject showed a longer P-wave duration. This subject experienced a first documented AF episode 9.7 months after admission. Furthermore, neither P-wave SAECG vector magnitude duration nor $P$-wave duration on 12-lead surface ECG correlated to LAD assessed on admission $(r=0.14 ; P=0.16$, and $r=0.08 ; P=0.28$, respectively). Also, frequency domain analysis of $P$-wave SAECG vector magnitude did not demonstrate a statistically significant predictive value for first AF during follow-up (data not shown). These findings lead us to speculate that P-wave SAECG may be a useful tool for assessing AF regarding either short-term recurrence or short-term follow-up in subjects with abnormal P-wave duration.

Atrial fibrillation is a prevalent arrhythmia in Chagas' disease regardless of the severity of heart involvement, exhibiting an annual incidence comparable to that observed in the general elderly population. Electrocardiographic (frequent PSVC and PVC, ventricular couplets and VT episodes during 24-h ambulatory ECG monitoring), and echocardiographic (left atrial diameter and pulmonary arterial hypertension) variables demonstrate a long-term predictive value for atrial fibrillation in subjects with Chagas' disease. Additionally, the rate of both LV mass enlargement and LV systolic function deterioration during followup impacts the incidence of atrial fibrillation in this population.

\section{Limitations of the study}

One possible limitation of this investigation is that it was performed on a hospital-derived cohort referred to the 
arrhythmia unit with complaints of palpitation, perhaps limiting the extension of the present findings to a general, unselected population with Chagas' disease.

Optimal normality thresholds for continuous variables were defined by ROC curve analyses. The cut-off value for LAD was lower than conventionally defined thresholds in other forms of cardiomyopathy. A direct extension of the present findings to clinical practice still awaits confirmation.

Another potential limitation is the small number of subjects enrolled. However, considering the strict followup of subjects with Chagas' disease in the present cohort and the number of first AF episodes observed, overall post hoc power analysis achieved high statistical power, validating the present results.

The present study aimed at investigating predictive factors impacting the occurrence of first AF lasting $>24 \mathrm{~h}$ in long-term follow-up of Chagas' disease patients. It was not the objective of the study to assess the impact of restoration of sinus rhythm after occurrence of AF on LV function and survival. We consider, however, that long-term maintenance of sinus rhythm may benefit survival, although this still requires confirmation. Furthermore, although a rather balanced distribution of both systemic arterial hyperten- sion and drugs prescribed according to first AF occurrence was observed at the end of follow-up, we cannot exclude a potential beneficial effect of blood pressure controlling drugs on first $A F$ incidence in this population.

A routine 12-lead ECG was performed during each clinical visit, every three to six months, representing the care taken to detect AF episodes during follow-up. Nevertheless, short-lasting and oligosymptomatic paroxysmal episodes of AF may have occurred in some subjects between clinical visits and may have been undetected before the persistent one was detected.

\section{Acknowledgments}

We are grateful to Adriano de Moraes, MD, and to Marcia B. Castier, MD, $\mathrm{PhD}$, for their support in carrying out electrocardiographic and echocardiographic examinations on admission, to Francisco M. Albanesi Filho, MD, and to Silvia H.C. Boghossian, MD, for their support in the follow-up of the subjects, and to Dr. Eduardo C. Barbosa, Dr. Alfredo S. Bomfim and Dr. Gustavo S. Duque for their observations, all from Hospital Universitário Pedro Ernesto, Universidade do Estado do Rio de Janeiro, Rio de Janeiro, RJ, Brazil.

\section{References}

1. Gersh BJ, Tsang TS, Seward JB. The changing epidemiology and natural history of nonvalvular atrial fibrillation: clinical implications. Trans Am Clin Climatol Assoc 2004; 115: 149-160.

2. Page RL. Clinical practice. Newly diagnosed atrial fibrillation. N Engl J Med 2004; 351: 2408-2416.

3. Marin Neto JA, Simoes MV, Sarabanda AV. Chagas' heart disease. Arq Bras Cardiol 1999; 72: 247-280.

4. Silva AS, Carvalho ME, Rodrigues VLCC. Doença de Chagas (Guide to Health care Professionals - The Government of the State of São Paulo, Brazil) (in Portuguese). Guia para profisionais da saúde. Governo do Estado de São Paulo [http://www.sucen.sp.gov.br/doencas/chagas/ texto chagas pro.htm]; 2008.

5. Brazil Federal Government. Guide of Diseases. Brazil Federal Government Official Report on Chagas' Disease. Brazil Federal Government [http://portal.saude.gov.br/portal/ svs/visualizar_texto.cfm?idtxt=21907]; 2004.

6. Maguire JH, Hoff R, Sherlock I, Guimaraes AC, Sleigh AC, Ramos NB, et al. Cardiac morbidity and mortality due to Chagas' disease: prospective electrocardiographic study of a Brazilian community. Circulation 1987; 75: 1140-1145.

7. Gazin P, Melo G, Abuquerque A, Oliveira W Jr, Soula G, Audry $P$. Chagas disease in a rural area of Northeast Brazil. Bull Soc Pathol Exot 2004; 97: 189-192.
8. Benchimol-Barbosa PR. The oral transmission of Chagas' disease: an acute form of infection responsible for regional outbreaks. Int J Cardiol 2006; 112: 132-133.

9. Hagar JM, Rahimtoola SH. Chagas' heart disease. Curr Probl Cardiol 1995; 20: 825-924.

10. Espinosa RA, Pericchi LR, Carrasco HA, Escalante A, Martinez O, Gonzalez R. Prognostic indicators of chronic chagasic cardiopathy. Int J Cardiol 1991; 30: 195-202.

11. Bestetti RB, Dalbo CM, Freitas OC, Teno LA, Castilho OT, Oliveira JS. Noninvasive predictors of mortality for patients with Chagas' heart disease: a multivariate stepwise logistic regression study. Cardiology 1994; 84: 261-267.

12. Carrasco HA, Parada H, Guerrero L, Duque M, Duran D, Molina C. Prognostic implications of clinical, electrocardiographic and hemodynamic findings in chronic Chagas' disease. Int J Cardiol 1994; 43: 27-38.

13. Bestetti RB, Dalbo CM, Arruda CA, Correia FD, Freitas OC. Predictors of sudden cardiac death for patients with Chagas' disease: a hospital-derived cohort study. Cardiology 1996; 87: 481-487.

14. Prata SP, da Cunha DF, da Cunha SF, Prata SC, Nogueira N. Prevalence of electrocardiographic abnormalities in 2,000 aged and non-aged chagasic patients. Arq Bras Cardiol 1993; 60: 369-372.

15. Schocken DD, Arrieta MI, Leaverton PE, Ross EA. Preva- 
lence and mortality rate of congestive heart failure in the United States. J Am Coll Cardiol 1992; 20: 301-306.

16. Acquatella $H$, Schiller NB, Puigbo JJ, Giordano $H$, Suarez $\mathrm{JA}$, Casal $\mathrm{H}$, et al. M-mode and two-dimensional echocardiography in chronic Chagas' heart disease. A clinical and pathologic study. Circulation 1980; 62: 787-799.

17. Lang RM, Bierig M, Devereux RB, Flachskampf FA, Foster E, Pellikka PA, et al. Recommendations for chamber quantification: a report from the American Society of Echocardiography's Guidelines and Standards Committee and the Chamber Quantification Writing Group, developed in conjunction with the European Association of Echocardiography, a branch of the European Society of Cardiology. J Am Soc Echocardiogr 2005; 18: 1440-1463.

18. Benchimol-Barbosa PR, de Souza Bomfim A, Barbosa EC, Ginefra P, Helena Cardoso Boghossian S, Destro C, et al. Spectral turbulence analysis of the signal-averaged electrocardiogram of the atrial activation as predictor of recurrence of idiopathic and persistent atrial fibrillation. Int J Cardiol 2006; 107: 307-316.

19. Abe R, Nishida T. The criteria for the prediction of paroxysmal atrial fibrillation by time domain analysis of the $\mathrm{P}$ wavetriggered signal-averaged electrocardiogram. Nippon Rinsho 1995; 53: 496-502.

20. Fieiss JL. Statistical methods for rates and proportions. 2nd edn. New York: Wiley; 1981.

21. Benchimol-Barbosa PR, Barbosa-Filho J. Atrial mechanical remodeling and new onset atrial fibrillation in chronic Chagas' heart disease. Int J Cardiol 2008; 127: e113-e115.

22. Bestetti RB, Freitas OC, Muccillo G, Oliveira JS. Clinical and morphological characteristics associated with sudden cardiac death in patients with Chagas' disease. Eur Heart $J$ 1993; 14: 1610-1614.

23. Dias JC, Kloetzel K. The prognostic value of the electrocardiographic features of chronic Chagas' disease. Rev Inst Med Trop São Paulo 1968; 10: 158-162.

24. Teixeira AR, Nascimento RJ, Sturm NR. Evolution and pathology in Chagas disease - a review. Mem Inst Oswaldo Cruz 2006; 101: 463-491.

25. Viotti R, Vigliano C, Lococo B, Bertocchi G, Petti M, Alvarez $M G$, et al. Long-term cardiac outcomes of treating chronic Chagas disease with benznidazole versus no treatment: a nonrandomized trial. Ann Intern Med 2006; 144: 724-734.

26. Turhan $\mathrm{H}$, Yetkin $\mathrm{E}$, Atak R, Altinok $\mathrm{T}$, Senen $\mathrm{K}$, lleri $\mathrm{M}$, et al. Increased $\mathrm{p}$-wave duration and $\mathrm{p}$-wave dispersion in patients with aortic stenosis. Ann Noninvasive Electrocardiol 2003; 8: 18-21.

27. Benchimol-Barbosa PR, Barbosa-Filho J. Comment on the article by Vazquez and Marquez-Garcia. High-risk vs. low- risk for thrombo-embolic events: who are in the intermediate-risk group? What should be done then? Int J Cardiol 2008; 124: 358-359.

28. Duytschaever M, Haerynck F, Tavernier R, Jordaens L. Factors influencing long term persistence of sinus rhythm after a first electrical cardioversion for atrial fibrillation. Pacing Clin Electrophysiol 1998; 21: 284-287.

29. Rosenheck $S$. Signal-averaged $P$ wave in patients with paroxysmal atrial fibrillation. Pacing Clin Electrophysiol 1997; 20: 2577-2586.

30. Funk M, Richards SB, Desjardins J, Bebon C, Wilcox H. Incidence, timing, symptoms, and risk factors for atrial fibrillation after cardiac surgery. Am J Crit Care 2003; 12: 424433.

31. Ianni BM, Mady C, Arteaga E, Fernandes F. Cardiovascular diseases observed during follow-up of a group of patients with undetermined form of Chagas' disease. Arq Bras Cardiol 1998; 71: 21-24.

32. Falk RH. Atrial fibrillation. N Engl J Med 2001; 344: 10671078.

33. Rassi A Jr, Rassi A, Little WC, Xavier SS, Rassi SG, Rassi $A G$, et al. Development and validation of a risk score for predicting death in Chagas' heart disease. N Engl J Med 2006; 355: 799-808.

34. Carod-Artal FJ, Vargas AP, Horan TA, Nunes LG. Chagasic cardiomyopathy is independently associated with ischemic stroke in Chagas disease. Stroke 2005; 36: 965-970.

35. Bestetti R. Stroke in a hospital-derived cohort of patients with chronic Chagas' disease. Acta Cardiol 2000; 55: 33-38.

36. Gentlesk PJ, Sauer WH, Gerstenfeld EP, Lin D, Dixit S, Zado $E$, et al. Reversal of left ventricular dysfunction following ablation of atrial fibrillation. J Cardiovasc Electrophysiol 2007; 18: 9-14.

37. Benchimol Barbosa PR. Noninvasive prognostic markers for cardiac death and ventricular arrhythmia in long-term follow-up of subjects with chronic Chagas' disease. Braz J Med Biol Res 2007; 40: 167-178.

38. Benchimol-Barbosa PR. Cardiac remodeling and predictors for cardiac death in long-term follow-up of subjects with chronic Chagas' heart disease: a mathematical model for progression of myocardial damage. Int J Cardiol 2009; 131: 435-438

39. Barbosa FJ, Albanese Filho FM, Barbosa PR, Lacerda RC, Ginefra P. Electrocardiogram in dilated cardiomyopathy. Rev Port Cardiol 1989; 8: 769-774.

40. Ehrlich JR, Zhang GQ, Israel CW, Hohnloser SH. P-wave signal averaging-ECG: normal values and reproducibility. $Z$ Kardiol 2001; 90: 170-176. 